\title{
Cilia-powered particle streaming
}

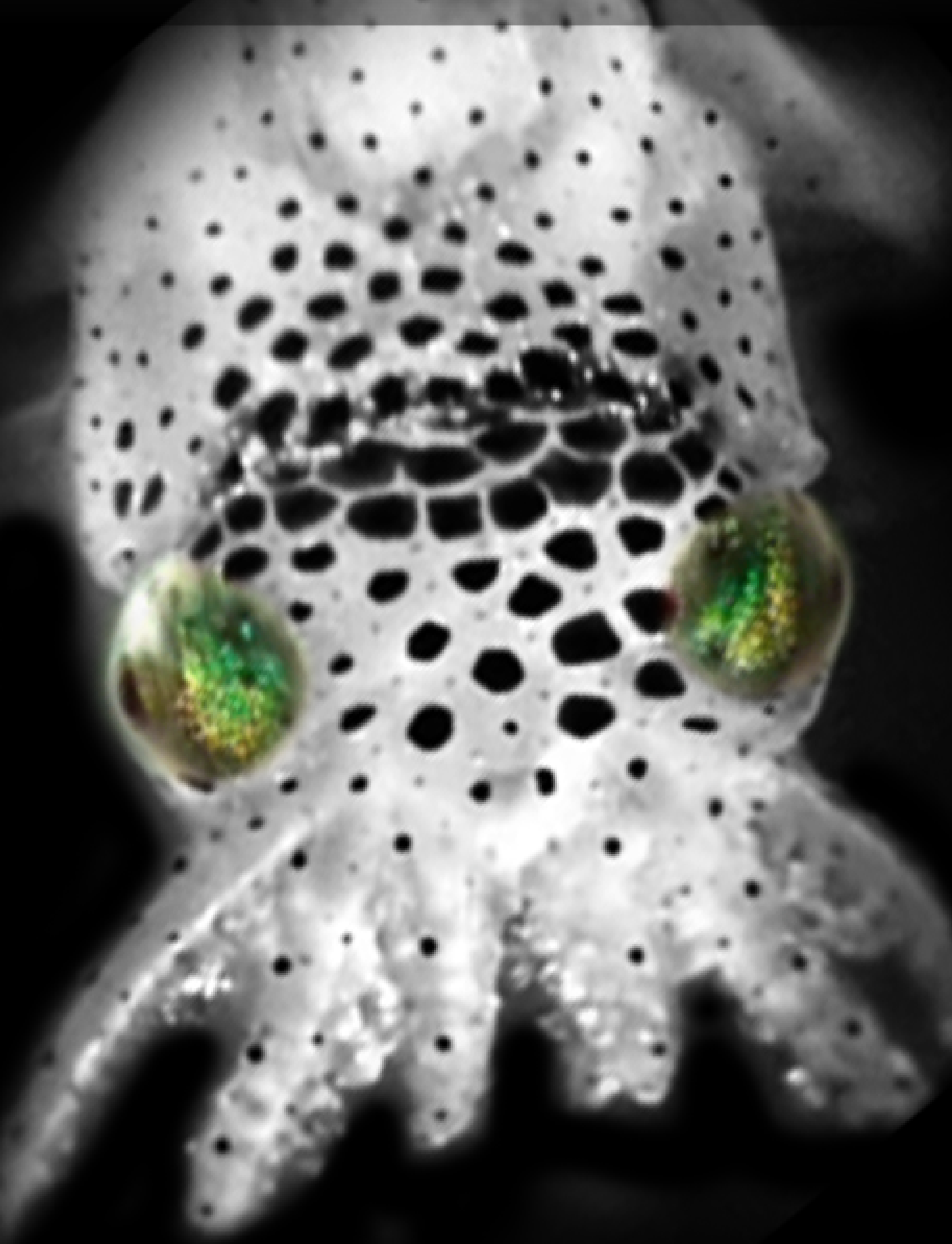

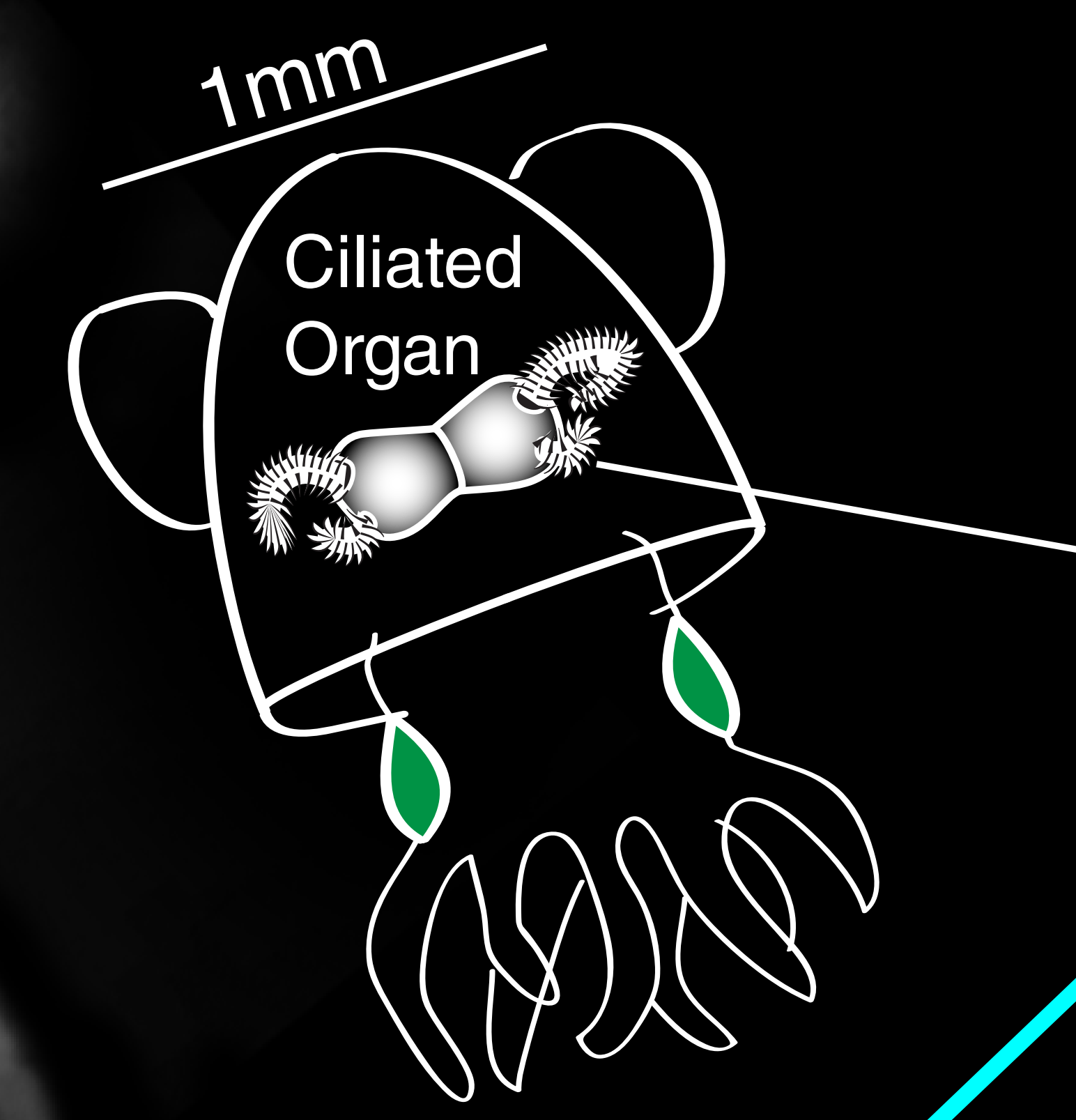

A look at the entire

cilia-generated flow field
Cilia kinematics and surface topology interact to create a complex flow pattern around the ciliated appendages, as visualized by particle path lines. This flow achieves

(1) sorting of bacteria-sized particles through viscous streaming

strong self-cleansing currents

(3) bacteria accumulation in between the appendages through flow stagnation

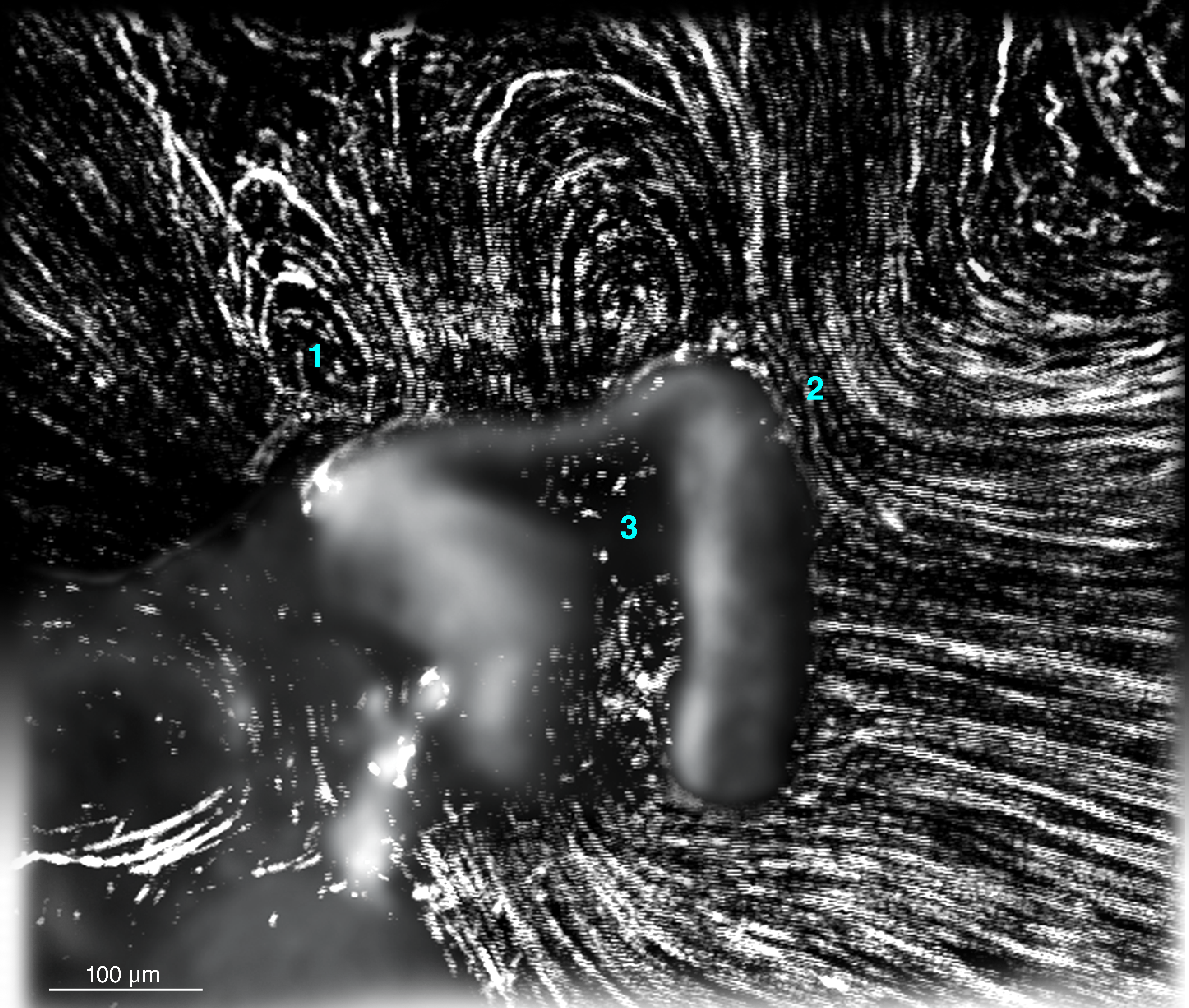

Ciliated Appendages

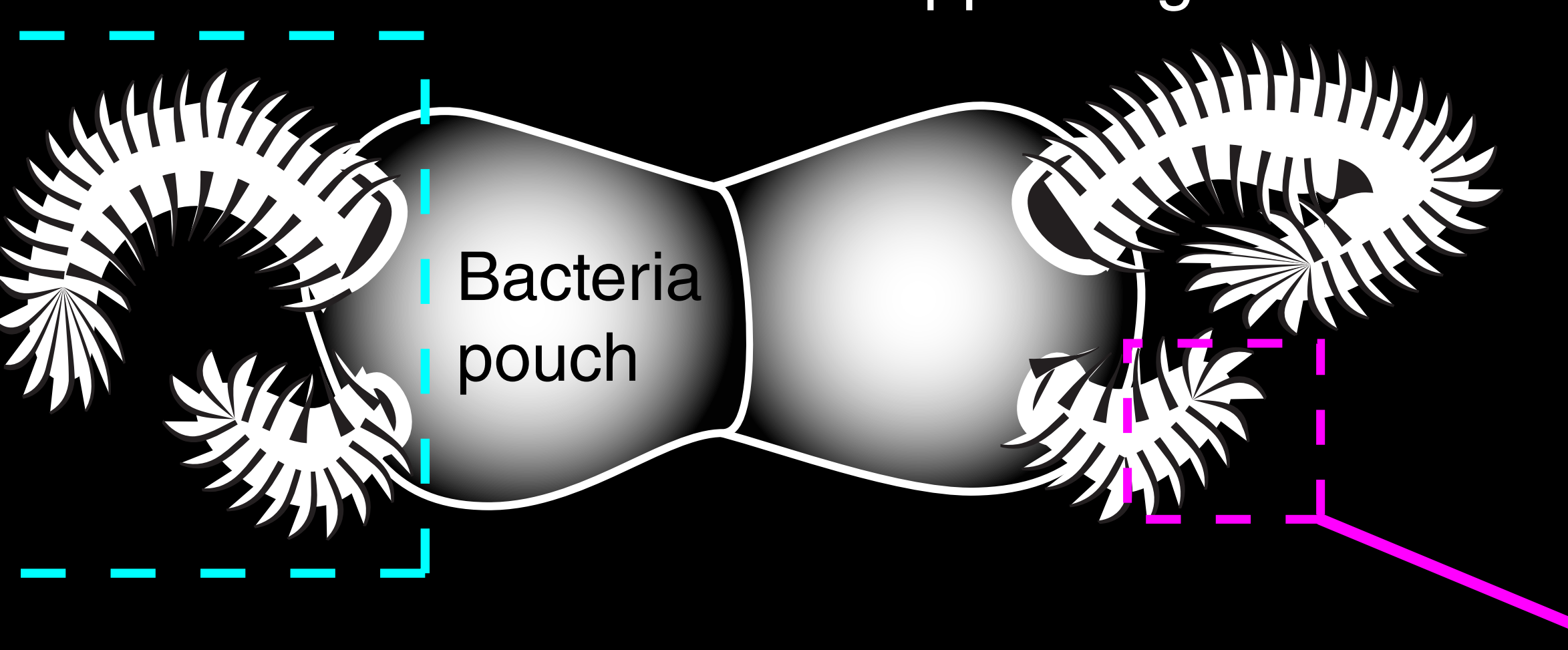

Close up on cilia-powered particle streaming

Viscous streaming is driven by surface oscillations. On ciliated surfaces, quasi-oscillatory ripples are achieved by spatiotemporal coordination of

ciliary beating: Stroke phases of neighboring cilia are slightly shifted, resulting in periodic waves whose crests and troughs are formed by cilia in the effective and recovery stroke, respectively. These so-called metachronal waves travel across the surface, as visualized by high-speed camera snapshots (left: top view; right: cross-section).

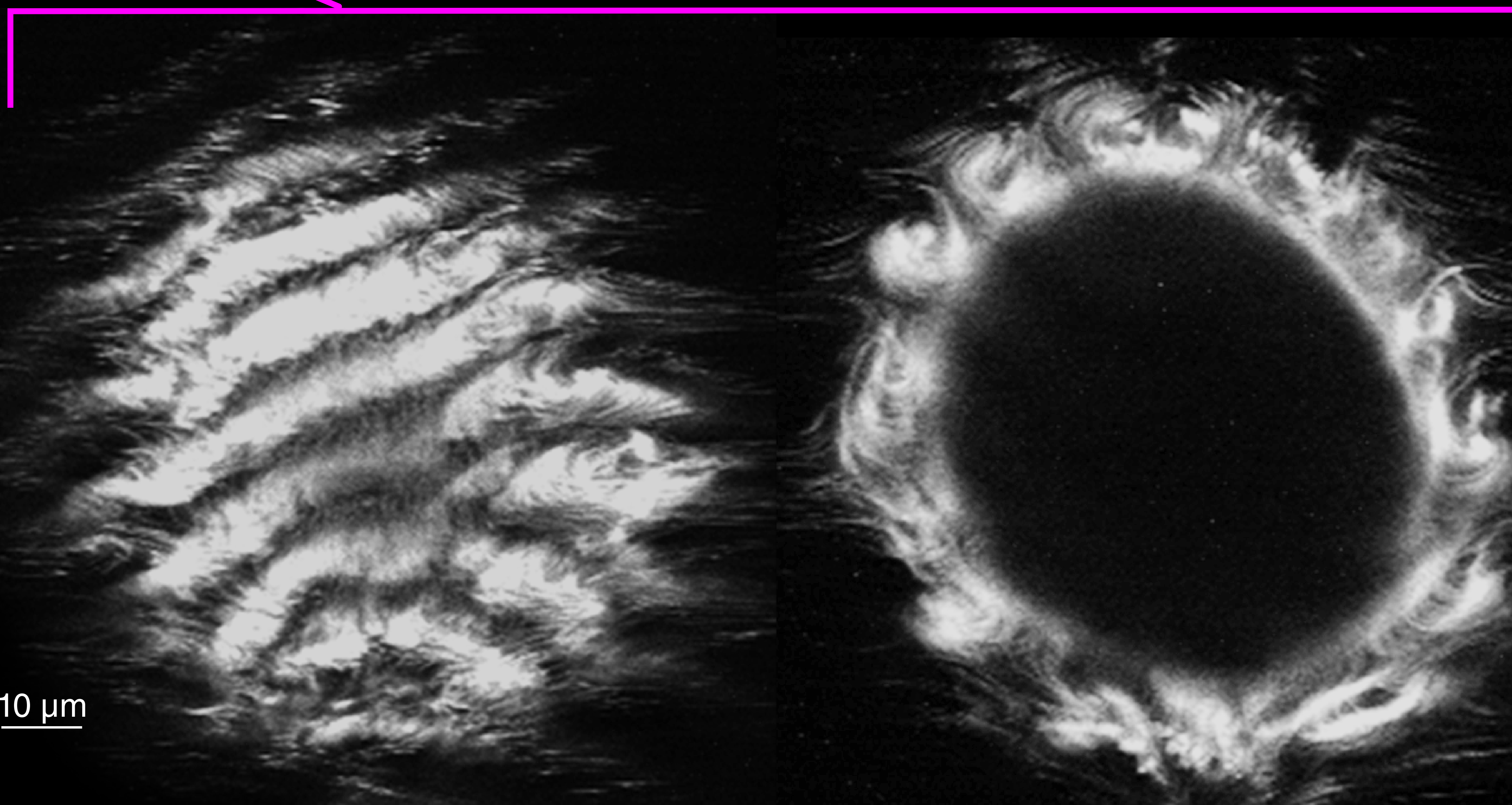

The metachronal wave generate vortical flows capable of accumulating particles: some (larger) particles cross streamlines and are diverted to the center whereas others (smaller) particles follow an outer trajectory, as visualized by particle path lines.
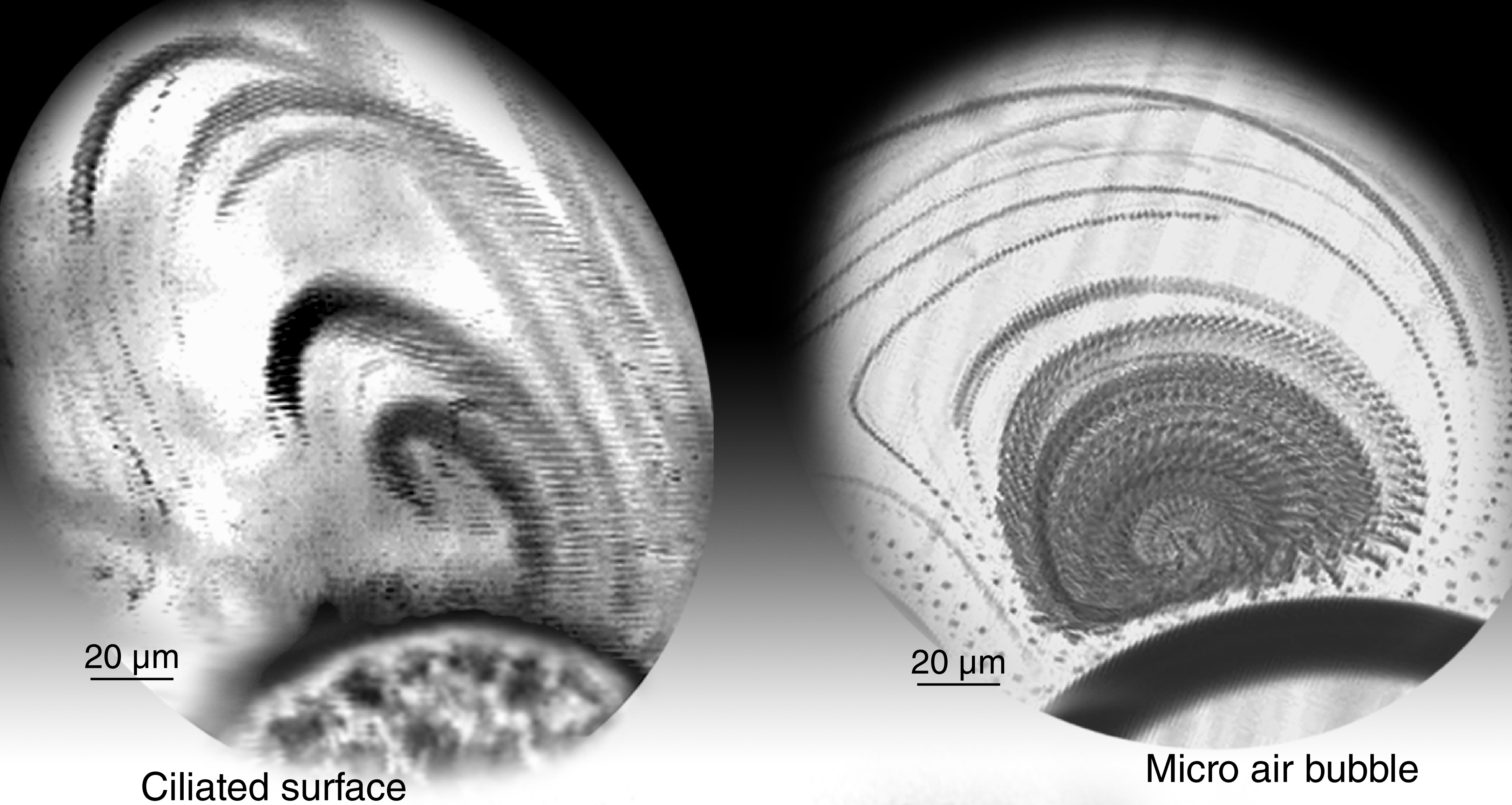

with streaming function can be produced with other oscillating surfaces at low Reynolds numbers, such as vibrating micro air bubbles in liquid.

Micro air bubble waves can be visualized using streak lines. Here, the ciliated organ, which contains squid ink, was pierced to release a thin line of ink tracing the cilia-generated flow.

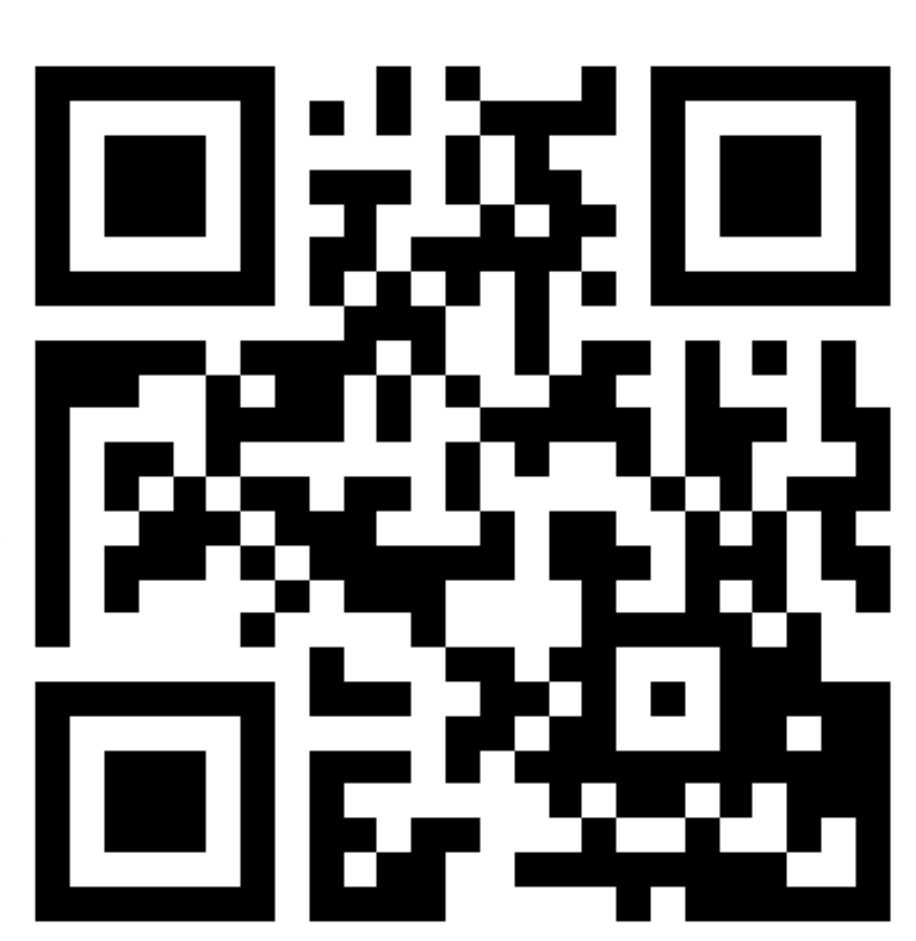

